\title{
LA FORMAZIONE DEGLI INSEGNANTI DI RELIGIONE NELL'UNIONE EUROPEA
}

\section{A formação dos professores de Ensino Religioso na União Européia}

Flavio Pajer ${ }^{1}$

\section{Resume}

Il processo di unificazione economica e politica dell'Europa - in atto ormai da quasi 50 anni se si considera il Trattato di Roma (1957) come l'evento fondante - non può e non deve produrre, almeno a breve termine, un livellamento delle istituzioni nazionali per uniformarle a un comune standard transnazionale. Questo è vero soprattutto per i vari sistemi educativi nazionali. Essi sono troppo ancorati alla storia e alla cultura delle rispettive nazioni, troppo diversi per lingua, per struttura e organizzazione, al punto che una loro armonizzazione risulterebbe un insulto allo specifico e irriducibile patrimonio culturale e storico delle varie nazioni europee.

Parole-chiavi: Patrimonio culturale; Educazione; Patrimonio storico; Europa.

\section{Resumo}

O processo de unificação econômica e política da Europa que está ocorrendo nestes quase cinquenta anos, considerando o Tratado de Roma (1957) como o evento fundador, não pode e não deve produzir, pelo menos breventemente, um nivelamento das instituições nacionais para uniformizar um estado comun transnacional. Sobretudo por existir os vários sistemas educacionais nas diferentes nações. Estes são sustentados na história e na diversidade cultural das respectivas nações, pelas diversas línguas, assim como pelas estruturas e organizações, ao ponte que uma harmonização resultará um insulto ao específico e irredutível patrimônio cultural e histórico das várias nações européias.

Palavras-chave: Patrimônio cultural; Educação; Patrimônio histórico; Europa.

1 Flavio PAJER, FSC, professore di Pedagogia e Didattica delle Religioni alla Pontificia Università Salesiana di Roma e alla Facoltà di Teologia dell'Italia Meridionale, Napoli; Presidente del Forum Europeo per l'istruzione religiosa nelle scuole pubbliche. Fratelli delle scuole Cristiane - Via Aurélia, 47600165 - Roma- Itália.

E-mail: fpajer@lasalle.org 


\section{L'Europa plurale}

Se poi si considera l'attuale "Europa dei 25 " dal punto di vista dell'insegnamento religioso praticato nelle scuole, altre importanti variabili intervengono a complicare il mosaico delle situazioni. Tra queste vanno ricordate:

- la singolare "geografia confessionale" del continente, che si presenta articolato in un Sud prevalentemente cattolico (Portogallo, Spagna, Francia, Belgio, Italia, Malta, Austria, cui vanno aggiunte l'Irlanda e la Polonia), in un Centro-Nord prevalentemente protestante (dal Regno Unito ai Paesi baltici, dalla Cekia ai paesi scandinavi), e in una regione orientale, di cultura slava e di tradizione ortodossa (Grecia, Cipro, Paesi balcani);

- la differente tipologia dei rapporti giuridici tra Stati e Chiese, che condizionano fortemente il profilo legale e contenutistico dell'insegnamento religioso: si va infatti dal modello separatista (tipico della Francia e della Slovenia, dove per legge non può esistere un insegnamento religioso propriamente detto nelle scuole pubbliche), al modello concordatario (una decina di Stati regolano tuttora l'insegnamento religioso in base a un accordo con le chiese cristiane, garantendo da una parte l'identità della propria tradizione religiosa ma al tempo stesso una certa neutralità statale, e soprattutto il diritto alla libertà religiosa di famiglie e alunni, secondo una corretta accezione di laicità), fino al modello integrazionista, tipico degli Stati che hanno contratto un legame storico privilegiato con una particolare Chiesa (il Regno Unito con la Chiesa anglicana, gli Stati scandinavi con le tradizione evangelico-luterana, la Grecia e Cipro con la Chiesa ortodossa: in questi casi la religione insegnata oggi come dimensione della cultura "nazionale" più che come catechesi - è talmente integrata con le diverse discipline scolastiche da diventare una materia ordinaria più o meno obbligatoria come le altre);

- la diversa articolazione, all'interno del sistema scolastico nazionale, tra scuole pubbliche, sia statali che comunali, e scuole libere confessionali gestite dalle varie chiese riconosciute dallo Stato (queste ultime, per esempio, coprono appena il 5\% della popolazione scolastica in Italia, ma salgono a oltre il 20\% in Francia, al 33\% in Spagna, per raggiungere il 60\% nel Belgio e il $70 \%$ nei Paesi Bassi, di cui metà cattoliche e metà protestanti);

- se si guardano i modelli di amministrazione e di funzionamento del sistema educativo, le differenze tra paese e paese sono altrettanto evidenti: si va da una gestione centralizzata in mano a un unico ministero dell'istruzione nazionale (Francia, Italia, Grecia, Polonia), a una gamma diversificata di amministrazioni regionali o locali che funzionano secondo il principio della sussidiarietà (per esempio, a livello dei 16 Länder in Germania, dei 26 cantoni in Svizzera [che però non fa parte dell'UE], delle 3 comunità linguistiche in 
Belgio, delle 15 autonomías in Spagna, del centinaio di contee nel Regno Unito ...);

- in particolare, pensando al profilo della materia "religione", va tenuto presente il diverso grado della base legale su cui si fonda (per es:, ha una base costituzionale in Germania, in Belgio o in Grecia, ma poggia solo su un concordato in molti altri casi, o è soggetto alla precarietà delle leggi parlamentari in altri casi ancora); di conseguenza, risulta diverso il grado di curricolarità della materia (può essere materia ordinaria e obbligatoria, ordinaria opzionale, o semplicemente facoltativa, o del tutto extra-curricolare); ed è importante ricordare che se nei paesi occidentali il principio della distinzione tra istruzione religiosa scolastica e catechesi ecclesiale è ormai generalmente condivisa anche ufficialmente dalle autorità religiose, non altrettanto si può dire dei paesi orientali usciti da pochi anni dall'egemonia comunista e con forte prevalenza di tradizione ortodossa.

E' facilmente intuibile, anche per un lettore non-europeo, che l'intreccio di queste variabili rende praticamente impossibile parlare al singolare della "formazione dell'insegnante di religione": i modelli di formazione sono tanti quanti sono i sistemi o i sotto-sistemi scolastici. Sono modelli difficilmente generalizzabili o esportabili dal contesto che li ha visti nascere ed evolvere. Bisognerebbe parlarne stato per stato, o chiesa per chiesa. L'Europa è stata, e resta, il continente delle cento culture regionali, un patchwork colorato dalle mille sfumature. In Europa c'è di tutto, eccetto che uniformità. Ma al fine di offrire qui uno sguardo sintetico, panoramico, mi limito a rilevare alcuni tratti comuni o maggiormente frequenti della figura dell'insegnante di religione "europeo" quanto alla sua formazione iniziale e alla formazione permanente.

\section{Le strutture della formazione iniziale}

1. La formazione di base degli insegnanti di religione cristiana, reclutati secondo la propria confessione di appartenenza, viene generalmente assicurata mediante due percorsi accademici:

- o in una università statale dotata di facoltà di teologia e/o di scienze dell'educazione: è il caso anzitutto della Germania (con una ventina di facoltà teologiche statali, con apposite Hochschule, in parte cattoliche e in parte protestanti), della Grecia (facoltà teologica ortodossa), del Regno Unito (dove ordinariamente ogni insegnante di religione, oltre al titolo per insegnare una data materia profana, deve aver conseguito un diploma in teologia o scienze religiose presso una università o un politecnico statale), della Danimarca (dove il curricolo accademico di "scienze religiose" è obbligatorio per qualsiasi insegnante della scuola primaria che voglia ottenere l'abilitazione 
all'insegnamento generale), della Norvegia, della Svezia, della Lituania, della Estonia, della Repubblica Ceca, dell' Ungheria (tutti paesi che, con varianti strutturali notevoli, offrono curricoli specifici di teologia e pedagogia religiosa in università oppure in istituti parificati di grado superiore);

- o in istituti superiori di scienze religiose, pedagogiche e/o catechetiche, gestiti dalle Chiese, ma riconosciuti idonei a rilasciare titoli abilitanti all'insegnamento nelle scuole pubbliche, sia statali che confessionali: è il sistema seguita in generale dai paesi a maggioranza cattolica (Austria, Belgio, Croazia, Irlanda, Italia, Lussemburgo, Malta, Polonia, Portogallo, Slovacchia, Spagna), ma anche dall'Olanda (a confessione mista), dalla Romania (a maggioranza ortodossa, paese preconizzato a entrare nell'UE nel 2007).

2. La durata degli studi di base può variare da un minimo di due anni accademici per i candidati che insegneranno nella scuola dell'obbligo, a un massimo di cinque anni per i futuri insegnanti di scuola secondaria. Nel caso della Germania, per esempio, sono previsti sei semestri accademici per poter insegnare nella scuola primaria, otto semestri per insegnare nel primo ciclo della secondaria, e dieci semestri per insegnare nelle superiori e nelle scuole professionali. In diversi paesi cattolici, dove la struttura privilegiata di formazione è l'istituto ecclesiastico di scienze religiose la durata è generalmente di tre anni di studi religiosi-pedagogici per poter insegnare nella scuola primaria, e quattro anni (ma cinque nel prossimo futuro) per insegnare nelle secondarie.

3. I piani di studio variano da un paese all'altro in ragione dell'organizzazione del curricolo, che può essere di tre tipi:

- programma integrato, nel quale gli studi accademici e quelli propriamente professionalizzanti sono indissociabili (per esempio: nei Paesi scandinavi, in Olanda e in alcuni Colleges of Higher Education britannici);

- programma parallelo, in cui gli studi delle discipline teologiche sono simultanei ma non correlati con quelli pedagogico-didattici (ciò si verifica in alcune istituzioni britanniche, in Lituania, in parte in Polonia);

- programma consecutivo, che prevede un primo ciclo di studio dei saperi accademici, e un secondo ciclo di studi professionali (diritto scolastico, didattica, metodologie applicative), comprendenti anche il tirocinio pratico (è la prassi seguita piuttosto in Germania e in Belgio, ma anche i diversi paesi cattolici seguono in genere questo standard).

4. Sui contenuti dei curricoli formativi è estremamente difficile render conto in modo dettagliato, anche perché, di fatto, esiste sempre un divario più o meno rilevante tra l'enunciato formale dei corsi (quelli presentati nel menù del Programma annuale, che devono adeguarsi ai criteri dettati dalla ratio studiorum ufficiale) e ciò che viene effettivamente insegnato e appreso nella didattica pratica. Appaiono costanti comunque almeno tre aree di saperi-competenze: 
- l'area dei saperi religionistici, includenti il fenomeno religioso di tutti tempi e quello odierno, la religione ebraico-cristiana, le altre religioni storiche; l'area comprende in linea generale: a) un approccio informativo e critico alle principali scienze della religione (come la storia, la fenomenologia, la psicologia, la sociologia, la linguistica... applicate alla religione); b) l'iniziazione alle classiche discipline ecclesiastiche (esegesi biblica, teologia, storia, etica, pastorale).

- l'area dei saperi antropologici, relativi alla condizione dell'alunno, alla società e alla cultura in cui vive, alla storia religiosa e al costume del paese, ai valori etici e simbolici presenti nel contesto;

- l'area delle competenze della comunicazione pedagogico-didattica propria del ciclo scolastico in cui il candidato eserciterà l'insegnamento, competenze includenti non solo le metodologie e le tecniche del saper-insegnare, ma anche le capacità metadidattiche, come quelle relazionali, gestionali, organizzative.

5. A proposito di quest'ultimo aspetto della formazione, dagli anni 90 si parla e si scrive spesso in Europa di "qualificazioni-chiäkdhe un buon insegnante di religione deve acquisire e saper esercitare, come la capacità di: prendere decisioni didattiche appropriate e tempestive, di discernere i problemi della condizione giovanile, di esprimere solidarietà senza essere demagogici o diplomatici, di collaborare efficacemente nel team dei docenti, di saper progettare pedagogicamente a breve e medio termine, di auto-verificare le proprie risorse e capacità didattiche, di assumere responsabilità sociali nell'istituzione scuola e fuori scuola, di saper giustificare pubblicamente le proprie scelte e opinioni personali, di saper dialogare e moderare discussioni e dibattiti tra giovani e tra giovani e adulti.

6. E' a partire dagli anni ' 80 che, specialmente nei paesi del centronord Europa, si sviluppa una didattica professionale della religione, un settore scientifico della più ampia pedagogia della religione: tale didattica vuol essere insieme una scienza ed un'arte, una disciplina accademica e una tecnica collaudata dalla reciprocità circolare tra teoria e prassi. Si raffinano così, in Germania ma non solo, diverse didattiche specialistiche, quali: la didattica della correlazione, la didattica del simbolo, la didattica estetica o attraverso l'arte, la didattica narrativa, la didattica biblica, la didattica ermeneutica, la didattica modulare, la didattica comparativa o del confronto; più recentemente, la didattica interculturale e interreligiosa, o dell' interreligious learning. Non cessa di aumentare la mole degli studi teorici e degli strumenti pratici per rendere plausibili e perfezionare questi diversi approcci, da considerarsi comunque complementari e mai esclusivi.

7. Un periodo di tirocinio pratico fa parte integrante della formazione di base del futuro insegnante; è anzi uno dei cardini qualificanti della 
preparazione professionale, che mai viene ritenuta acquisita con la fine degli esami teorici o con il solo diploma accademico. In genere, la stessa dissertazione finale non è accettata se non include almeno una parte contestuale di valutazione critica di un'esperienza didattica effettivamente progettata, eseguita ed analizzata dai vari punti di vista. E' la fase finale della formazione iniziale, detta anche "qualificazione sul lavoro": un periodo di transizione tra la formazione accademica e l'inserimento a pieno titolo nella attività professionale; è un periodo di valutazione formale delle capacità del candidato per attestarne le competenze professionali. Il tirocinio è organizzato in stretta collaborazione con la facoltà o l'istituto presso cui il candidato ha seguito la sua formazione. Il termine italiano tirocinio (etimologicamente, dal latino: attività del principiante) significa appunto preparazione pratica necessaria per esercitare un mestiere o una professione. Normalmente il tirocinio (detto Prakticum o Probezeit, cioè periodo di prova, in Germania e in Austria, Training nel Regno Unito e Irlanda, Stage oppure Initiation pratique in Belgio, Practicum in Spagna...) è condotto sotto la guida di un docente esperto in didattica disciplinare (chiamato tutor o teacher-tutor o ancora probation manager nei sistemi anglosassoni, mentor o fachleiter in Germania, inspecteur in Belgio, catecheta distrettuale in Olanda, religious counsellor a Malta, orientador de estágio in Portogallo...). Si tratta di figure istituzionali locali o regionali, che assolvono al duplice compito di guidare i candidati nel loro tirocinio in aula e di accompagnare poi i nuovi insegnanti nei loro primi anni di esperienza professionale. La durata del tirocinio può estendersi da poche settimane a due semestri, secondo gli statuti accademici dei paesi. In caso di valutazione negativa del tirocinio (i casi registrati restano comunque assai rari), il candidato se lui stesso lo richiede - può essere ammesso a ripetere la fase finale della qualificazione, oppure ripetere l'intero stage.

8. Idoneità. In molti sistemi nazionali, l'abilitazione professionale del candidato deve essere accompagnata dalla idoneità canonica. L'esito positivo del tirocinio è un requisito necessario ma non ancora sufficiente per l'immissione in ruolo del candidato nell'organico della scuola, almeno nella scuola secondaria. Mentre infatti nella scuola primaria l'insegnante di religione è lo stesso insegnante titolare di classe (che conserva comunque la facoltà di auto-esonerarsi dall'insegnamento religioso quando questo è di natura confessionale: è la cosiddetta clausola di coscienza), l'insegnante di religione nella scuola secondaria ha bisogno di una specifica licentia docendi da parte dell'autorità competente della sua chiesa o comunità religiosa di appartenenza. E' la dichiarazione di idoneità in vigore nei paesi cattolici, è la missio canonica per i cattolici nei paesi tedeschi, è la vocatio per gli evangelici tedeschi, è il certificato di battesimo per gli insegnanti appartenenti alle chiese ortodosse. Non è richiesta invece nessuna autorizzazione ecclesiastica per gli insegnanti anglicani e nem- 
meno per quelli luterani: nel Regno Unito e nei paesi scandinavi l'insegnante di religione è reclutato e assunto secondo parametri esclusivamente accademici e professionali.

Le chiese in questi paesi - trattandosi di nazioni che conservano nelle loro strutture statuali un certo carattere confessionale legato al rapporto privilegiato con una data chiesa storica - non hanno competenza giuridica sul reclutamento, sulla formazione e sulla assunzione degli insegnanti di religione. Si tratta d'altronde, in questi paesi, di un insegnamento legalmente transconfessionale o di multifaith religious education (Regno Unito), di insegnamento religioso 'oggettivo' o sulle religioni (Svezia), di insegnamento ecumenico tendenzialmente aconfessionale (Danimarca): forme tutte che non impegnano di per sé la soggettività confessionale dell'insegnante né quella dell'alunno.

9. E' evidente che esulano dal controllo delle autorità religiose gli insegnanti di etica naturale o di morale laica o di storia delle religioni, o di altre materie simili attivate come disciplina alternativa nel sistema scolastico del Belgio, della Germania, del Lussemburgo, dei tre paesi baltici (Estonia, Lettonia, Lituania), della Repubblica Ceca. Corsi opzionali aconfessionali sono attuati anche in una quindicina di "Scuole europee", a vocazione transnazionale, funzionanti in diversi paesi a servizio soprattutto dei figli degli imprenditori, impiegati o burocrati che lavorano in un paese estero ma in organismi europei. Il reclutamento come la formazione di questi insegnanti sono assicurati in genere dalle facoltà ordinarie di scienze umane gestite dallo Stato.

\section{La formazione permanente degli insegnanti di religione}

1. Anche sulle strutture e modalità della formazione permanente il panorama europeo si presenta molto vario ed eterogeneo. In generale si osserva questo: nei sistemi nazionali dove l'insegnante di religione gode di un chiaro statuto giuridico e professionale, egli ha la possibilità - anzi il dovere di frequentare periodicamente uno di quei centri o istituti regionali di aggiornamento pedagogico-didattico che organizzano corsi, seminari, sperimentazioni guidate, dibattiti con esperti, ricerche sul campo, simulazioni didattiche, visite culturali, esercizi di analisi e valutazione, ecc.

In Inghilterra operano i "centri regionali per insegnanti", gli istituti politecnici e la fondazione nazionale per la ricerca educativa: gli insegnanti di religione ne usufruiscono come i colleghi delle altre discipline. In Olanda funzionano tre Centri pedagogici (uno cattolico, uno protestante e uno laico) e i servizi regionali di consulenza scolastica. In Belgio intervengono il Segretariato nazionale della scuola cattolica e altri organismi misti che promuovono 
contatti fra insegnanti e ricercatori, fra scuola e università; fra questi l'Icafoc (Institut catholique pour la formation continue des professeurs des enseignements secondaires et supérieurs) che, nella sua sezione "Religione", organizza regolarmente giornate di riflessione e pubblica un periodico di informazione e scambio di progetti. In Baviera (Germania) funziona un istituto regionale (Akademie für Lehrerfortbildung), che ha il compito di rilevare i bisogni oggettivi e soggettivi, indicare le tematiche generali dell'aggiornamento, redigere il programma semestrale di attività, formare e aggiornare ispettori, dirigenti scolastici e formatori, predisporre materiali e sussidi anche in funzione dell'autoaggiornamento. Tutto ciò per gli insegnanti in generale e per le singole categorie, inclusi i docenti di religione.

In Danimarca e in Inghilterra, anche l'insegnante di religione può disporre, come tutti i suoi colleghi di altre discipline, di un semestre sabbatico da utilizzare per l'auto-aggiornamento, mentre un supplente lo sostituisce a scuola per tutto questo periodo.

2. Circa i contenuti presenti nei vari percorsi della formazione permanente, è abbastanza ovvio immaginare che, nella crisi globale e nei rapidi mutamenti di questi ultimi anni, certe tematiche sono diventate immancabilmente vere e proprie emergenze. Tre aree sono soprattutto sollecitate:

- il problema della rilegittimazione-ridefinizione dei contenuti culturali dei tradizionali programmi di religione: non c'è paese europeo che non abbia dovuto ridiscutere l'identità, le finalità, i contenuti e la didattica del corso di religione; gli insegnanti si sono trovati in prima fila in questo dibattito infinito e certamente incluso; essi si sono dovuti sottomettere a una verifica culturale e didattica assai generalizzata per poter far fronte alle impasses del vecchio profilo della disciplina e per sperimentare nuove vie ancora inesplorate. Sarebbe curioso ma interessante, per esempio, analizzare il lessico estremamente variegato e fluttuante, adottato nell'ultimo ventennio per connotare gli insegnamenti di religione, sulla religione. Si è parlato e scritto di insegnamento religioso confessionale, interconfessionale, ecumenico, cooperativo, transconfessionale, interreligioso, interculturale, storico-fenomenologico, biblico, etico, ermeneutico, antropologico-esperienziale, comparativo, narrativo, oggettivo, centrato su problemi, centrato su valori... A volte la stessa denominazione dei corsi di religione - quelli gestiti autonomamente dallo stato - ha preso nomi nuovi: "visioni della vita" (Olanda), "studio del fatto religioso" (Francia), "cultura religiosa" (in alcuni cantoni svizzeri), "Società cultura religione" (Spagna), "studio della vita, dell'etica, delle religioni" (Land del Brandeburgo, Germania)...;

- la ricerca e la sperimentazione in didattica disciplinare e interdisciplinare, nell'intento di adeguare il curricolo di religione agli standard organizzativi e didattici di una scuola primaria e secondaria ovunque in forte evolu- 
zione, scuola che domanda anche all'insegnante di religione nuove capacità meta-didattiche di diagnosi, di concertazione, di dialogo, di gestione e valutazione dell'atto didattico;

- la lettura e la ricomprensione della realtà psico-sociologica dell'alunno - credente o non credente - e del suo 'mondo vitale': ciò per aiutare l'insegnante a interpretare le nuove domande di senso provenienti dal mondo giovanile, a saper leggere l'esodo di molti dalle chiese e dal religioso tradizionale, a saper verificare le condizioni e le vie possibili di un'educazione religiosa aperta e dialogante in una società pluralista, in una cultura postcristiana, e attraverso una scuola che sempre più sembra espropriata del suo tradizionale ruolo educativo.

3. Rinnovo dei contenuti programmatici. E' del tutto evidente che queste aree emergenti di problemi che impegnano la formazione permanente non possono non rifluire sugli stessi orientamenti della formazione iniziale. E questo perché certi problemi che dapprima sono percepiti solo come un evento congiunturale, diventano spesso una dimensione strutturale della nuova cultura, e domandano quindi di ripensare da capo tutta l'architettura e l'articolazione della formazione fin dalla sua fase iniziale.

Di fatto, le condizioni culturali consigliano e impongono programmi di formazione diversi. Ecco, a titolo d'esempio, alcune tematiche che sono integrate in priorità $\mathrm{o}$ in sostituzione di altre tradizionali $\mathrm{o}$ da considerarsi obsolete: l'analisi delle pratiche formative (ricerca-azione), gli stages rivolti alle situazioni dei contesti ambientali; lo studio di casi e la risoluzione di problemi operativi; le problematiche che devono affrontare coloro che iniziano la professione nella scuola; l'organizzazione della formazione in rapporto alle precedenti esperienze ed ai progetti personali; la formazione in relazione alla ricerca sul campo; il lavoro di gruppo e la conoscenza psico-sociologica che lo riguarda...

4. Da più parti si sollecita un profilo rinnovato e qualitativamente più ricco della figura del futuro insegnante di religione. Tale profilo dovrebbe avere, idealmente, almeno queste componenti:

- acquisizione di una posizione teologica personale: l'attuale formazione teologica è criticabile un po' ovunque, perché si limita a fornire al candidato contenuti e metodi scientifici, rimandando però alla pratica l'applicazione e l'assimilazione di una personale posizione. Senza questa appropriazione personale, senza un'interpretazione antropologica contestualizzata, la teologia appresa rischia di restare una sovrastruttura. L'esperienza di molti formatori negli stages insegna che non pochi insegnanti sono incapaci di leggere e interpretare grandi fatti d'attualità alla luce dei principi teologici appresi. Per acquisire una posizione teologica personale sono importanti colloqui, dibattiti guidati in gruppo, esercizi di percezione e di proiezione, discorsi interdiscipli- 
nari, elaborazione monitorata di unità didattiche su tematiche centrali (quali: il problema di Dio, del male, della colpa; la creazione, il miracolo, fede-scienza, morte e resurrezione; l'identità personale...);

- la consapevolezza della propria funzione docente, superando le strategie della sopravvivenza rassegnata o di una fedeltà solo burocratica al proprio mestiere. L'insegnamento religioso, si sa, è estremamente dipendente dal gradimento degli studenti. "Nel periodo di tirocinio l'insegnante stenta spesso ad accettare il proprio ruolo di insegnante. Evita perciò le questioni difficili e non osa difendere il proprio parere contro quello degli altri, oppure affronta tematiche critiche della religione, o si dedica all'analisi scientifica di testi, o si perde in esposizioni altamente astratte; evita di dare voti bassi" (Udo Marenbach, direttore degli studi in una Hochschule tedesca);

- saper verificare le proprie capacità didattiche e la capacità di selezionare i metodi appropriati: ogni insegnante è diverso e la diversità va rispettata; ilo giovane insegnante va aiutato molto per conoscere e selezionare da una quantità illimitata di tecniche didattiche quelle che fanno al caso singolo della sua classe e della sua materia;

- la capacità di dialogare e moderare discussioni e dibattiti: è sempre molto esigente fare colloqui che risultino corretti e fecondi, in una "cultura liquida"come quella contemporanea, che incoraggia il dilagare incontrollato di opinioni accolte come verità, e di verità accettate come opinioni...;

- la competenza didattica deve esprimersi nella capacità di effettuare decisioni didattiche appropriate al soggetto, al gruppo, al contesto istituzionale, al momento cronologico e psicologico.

Una particolare insistenza si è notata ultimamente anche sul piano di una spiritualità specifica dell'insegnante di religione.

\section{Problemi aperti e sfide educative da affrontare nella scuola europea}

Motivi di preoccupazione, fonti di incertezza, sintomi di malessere se non di crisi sono all'ordine del giorno. A molte diagnosi, anche corrette e spietate, non corrispondono spesso terapie appropriate.

- C'è l'urgenza di formare insegnanti competenti nella didattica interreligiosa. Ascoltiamo, tra i tanti, uno studioso cattolico tedesco, che lavora proprio nella formazione teologica degli insegnanti (ENGLERT, 2004, 39-52). Parla con preoccupazione dei suoi studenti d'oggi, dei futuri insegnanti di religione che sta formando in particolare alla didattica interreligiosa: spesso dice - hanno soltanto conoscenze rudimentali persino della propria religione, conoscono la Bibbia in termini superficiali e acritici, probabilmente non si 
sono mai confrontati seriamente con il problema della fede e alcuni di essi hanno ben poca o nessuna esperienza di vita ecclesiale. Il problema è: che cosa capiterà quando questi studenti di oggi, diventati insegnanti, dovranno far conoscere a fanciulli e giovani anche le religioni non cristiane e renderli capaci di praticare il dialogo interreligioso? Un insegnamento religioso che si estende a diverse religioni, non richiede forse dall'insegnante che abbia almeno una solida familiarità con i testi delle fonti, della storia e della teologia di queste religioni? Se la pedagogia religiosa intende realmente attuare le sue ambizioni nella direzione dell'apprendimento interreligioso, non le resterà altra via d'uscita che quella di organizzare, in collaborazione con rappresentanti di altre religioni, un programma formativo corrispondente (MICHIELSSENS, 2005, p.12). Attualmente la pedagogia religiosa - afferma il nostro esperto - è ancora lontana da ciò. Ci sono singole iniziative, circoscritte, ma in generale il confronto con le altre religioni, ad eccezione forse dell'ebraismo, non ha finora un posto di rilievo. D'altra parte, come introdurre nuovi e consistenti elementi di studio in scienze religiose, senza gonfiare a dismisura l'attuale contingente di crediti che sono richiesti dallo studio della teologia, o senza cancellare altri importanti settori di studio? Problemi che rimangono aperti.

- E' indubitabile che i curricoli classici della formazione docente, creati sullo stampo della ratio studiorum imposta dalle autorità vaticane alle facoltà teologiche, riflettono ancora, più o meno direttamente, l'ideale della formazione seminaristica post-tridentina. I programmi di molti istituti di scienze religiose formano ancora i futuri insegnanti a insegnare la religione come se il pubblico di studenti fosse tutto composto di giovani credenti, o come se i bambini d'oggi, sia pur battezzati nella quasi totalità, avessero davanti una normale adolescenza di praticanti e potessero arrivare automaticamente a una normale maturità religiosa. E' in crisi il concetto stesso di "trasmissione del patrimonio religioso" alla nuova generazione. La stessa pedagogia profana ha dovuto rivoluzionare procedure, linguaggi, tecniche, ritmi di apprendimento. La pedagogia religiosa insegue con affanno, se non con sospetto, queste novità. E anche quando si introducono innovazioni, il successo non è mai garantito in partenza, perché il più delle volte ci si limita "a versare vino nuovo in una botte vecchia". Anche la pedagogia religiosa conosce e adotta, per esempio, le teorie del costruttivismo, del cognitivismo, della non-direttività, dell'auto-sviluppo, dell'educazione sistemi$\mathrm{ca} . .$. , ma con risultati più o meno problematici se non deludenti, nella misura in cui l'educazione propriamente religiosa sfugge, almeno in quello che ha di specifico e di irriducibile, ai parametri della sola trasmissione culturale, della socializzazione rituale o dell'imbonimento etico.

- "Chi deve insegnare religione?", si chiede un documento redatto da una commissione di Saggi incaricati nel 2002 dall'allora presidente della Commissione europea Romano Prodi di studiare le strategie di un dialogo tra le 
culture del Mediterraneo. Una distinzione iniziale è d'obbligo: bisogna evitare di confondere la proposta di una fede religiosa come l'intendono i fedeli di una religione, cioè come un catechismo, con l'insegnamento comparativo delle religioni, che si propone unicamente la conoscenza del fatto religioso e della sua storia. "E' quest'ultimo, e solo quest'ultimo, che costituisce una delle basi dell'apprendimento del dialogo delle culture attraverso l'educazione. L'insegnamento comparativo delle religioni deve essere affidato a dei professionisti dell'educazione, capaci, al di là delle loro opzioni di coscienza, di trasmettere una analisi comparata delle religioni con l'obbiettività dell'esperto e non con la passione dell'adepto". Il problema chiave è dunque quello della formazione di questi insegnanti. Che cosa propongono i "Saggi" delle culture mediterranee? "Proponiamo la creazione di una rete di istituzioni universitarie delle due rive del Mediterraneo, che organizzino delle sessioni di formazione degli insegnanti delle scuole primarie e secondarie in vista di abilitarli alla analisi comparativa delle materie (comprese le religioni) che contribuiscano alla costruzione dell'identità culturale, come pensiamo anche a sessioni di sensibilizzazione alla pedagogia della conoscenza reciproca e alle tecniche del dialogo interpersonale (...)." L'utopia è seducente, ma, come dice un vecchio proverbio italiano, "tra il dire e il fare c'è di mezzo il mare!".

- L'Europa finora non ha praticamente formato se non insegnanti di religione cristiana, a parte la quota estremamente minoritaria di insegnanti di "etica non confessionale" o di storia delle religioni. Ora la società europea si sta popolando di frazioni crescenti di cittadini non cristiani: sia perché appartenenti ad altre fedi, specie all'islam per via dell'immigrazione, sia perché non appartengono proprio più ad alcuna fede (circa il 20\% degli europei dichiara di non appartenere ad alcuna chiesa o organizzazione religiosa). Come potrà la scuola soddisfare i diritti educativi di questa crescente popolazione noncristiana? Se è già problematico formare un insegnante di religione che sappia educare alunni "culturalmente cristiani", quale insegnante di religione potrà/ dovrà parlare agli alunni non-cristiani? Problema apertissimo, pensando anche solo al caso degli insegnanti islamici: in diversi Stati (Gran Bretagna, Germania, Belgio, Spagna) sono istituiti legalmente o possono essere istituiti in futuro corsi di religione islamica, ma mancano gli insegnanti abilitati, perché non esistono istituzioni accademiche che possano assicurare la preparazione teologica e pedagogica dei candidati-imam. Ma problema apertissimo anche in un altro senso: quello degli insegnanti "cristiani" che non possono più assolutamente limitarsi a conoscere la propria religione e a insegnarla in modo autoreferenziale; come passaggio minimo, ma di capitale importanza, bisognerà aprire sempre più l'insegnamento mono-confessionale alle dimensioni degli altri due monoteismi. Con tutte le ovvie conseguenze sulla nuova formazione di cui avranno bisogno questi nuovi insegnanti. 


\section{Una guida bibliografica essenziale}

Per situare il problema dell'istruzione religiosa e del suo insegnante nell'Europa d'oggi è necessario aver presente il complesso quadro giuridico dei rapporti tra Stati e Chiese, perché solo a partire da questo mosaico di diversi assetti istituzionali e dalle differenti storie religiose che li hanno originati, si può evitare di cadere in facili e improprie generalizzazioni: Gerhard Robbers (ed.), Stato e Chiesa nell'Unione Europea, Nomos VerlagsgesellschaftGiuffrè editore, Milano 1996; Marco Ventura, La laicità dell’Unione Europea. Diritti, mercato, religione, Giappichelli editore, Torino 2001; Silvio FerrariIván C.Ibán, Diritto e religionein Europa occidentale, Il Mulino, Bologna_2004; Silvio Ferrari-W.Cole Durham-Elizabeth A.Sewell (eds.), Diritto e religione nell'Europa post-comunista, Il Mulino, Bologna 2004; José Luis Martinez-López-Muñiz (ed.), Religious Education in public schools (provisional edition pro manuscripto), Universidad de Valladolid (Derecho administrativo) 2003; Jean-Paul WILLAIME, Europe et religions. Les enjeux du XXIe siècle, Fayard, Paris 2004 .

Sui sistemi educativi europei e sulla formazione dell'insegnante in generale: Unità europea di Eurydice, La professione docente in Europa. Formazione iniziale e passaggio alla vita professionale, Le Monnier, Firenze 2003 (www.eurydice.org); Gianfranco Rescalli, Il cambiamento nei sistemi educativi. Processi di riforma e modelli europei a confronto, La Nuova Italia, Firenze 1995; ID, La scuola privata nell'Unione europea. Esperienze a confronto, La Nuova Italia, Firenze 1999; Flavio Pajer, La formazione degli insegnanti in Europa, Rivista Lasalliana, 2004/4, p.261-279.

Per farsi un'idea panoramica della tipologia degli insegnamenti di religione attualmente attivati nei sistemi educativi europei, mi permetto rinviare alla mia rassegna L'insegnamento scolastico della religione nella nuova Europa, Elledici, Leumann-Torino 1991, pp. 540, che ho più volte aggiornato con articoli sintetici, l'ultimo dei quali è Scuola e istruzione religiosa. Nuova cittadinanza europea, "Il Regno-attualità" 15 dicembre 2002, pp.774-788, tradotto in brasiliano in "Educação em Movimento", vol.II, n.5, suplemento, maioagosto 2003, pp.21-45. In particolare sull'insegnamento universitario delle scienze religiose e teologiche in Europa, vedi il numero monografico di "Religioni e società" (dell'Università di Firenze), a cura di F.Pajer, 2000/2, n.37, pp.3126, e il numero monografico di "Quaderni di diritto e politica ecclesiastica", a cura di A.Zanotti, 2002/1, pp.3-211. Per una informazione periodica e gratuita sulla attualità, sui documenti, sulle ricerche in corso, sulla bibliografia europea, cfr. il notiziario trimestrale plurilingue EuForNews del Forum europeo dell'istruzione religiosa, diffuso unicamente online (è possibile richiederlo al seguente indirizzo: fpajer@lasalle.org). 
Non mancano documenti recenti delle Chiese cristiane europee sul problema dell'insegnamento religioso in generale e specificamente sull'insegnante, a livello continentale ma più spesso a livello di chiese nazionali: CONSIGLIO DELLE CONFERENZE EPISCOPALI EUROPEE, L'insegnamento della religione cattolica nella scuola pubblica dei paesi europei, Elledici, Leumann-Torino 1991 (atti del Simposio europeo); CONFERENZA EPISCOPALE ITALIANA, Insegnare religione cattolica oggi, Nota pastorale, Roma 1991; ID., Gli Istituti di scienze religiose a servizio della fede e della cultura, Nota illustrativa e normativa del Comitato per gli ISR, Roma 1993; KIRCHENAMT DER EKD (Chiese evangeliche tedesche), Identität und Verständigung. Standort und Perspektiven des Religion sunterricht in der Pluralität, EKD vl., Gütersloh 1994; DIE DEUTSCHEN BISCHÖFE, Die bildende Kraft des Religionsunterricht. Zur Konfessionalität des katholischen RU, Sekretariat Der Deutschen Bischofskonferenz, 1996; COMISIÓN EPISCOPAL DE ENSEÑANZA Y CATEQUESIS, El Professor de Religión Católica, enero de 1998, Edice Madrid; COMITE MIXTE CATHOLIQUE-PROTESTANT, Eglises et laïcité en France. Etudes et propositions, Bayard-Centurion, Paris 1998; BISHOPS' CONFERENCE OF ENGLAND AND WALES, Statement on Religious Education in Catholic Schools, $5^{\text {th }}$ May 2000, Matthew James Publishing Ltd, Chelmsford (Essex), 2000; SECRETARIADO DE LA COMISIÓN EPISCOPAL DE ENSEÑANZA, Plan de formación sistemática complementaria. Profesorado de Religión católica, Edice, Madrid 2001.

Una attenzione sempre più insistente al problema dell'educazione religiosa pubblica dimostrano diversi organismi dell'Unione Europea e del Consiglio d'Europa, oltre che da autorità politiche e da intellettuali di singoli Stati: cf. i quattro Seminari di studio organizzati dal Commissario europeo ai Diritti dell'uomo del Consiglio d'Europa, Alvaro Gil-Robles, a Siracusa, Lovanio, Strasburgo e Malta, dal 2000 al 2004: www.commissioner.coe.int; commissioner.humanrights@coe.int, allo scopo di approfondire con esperti e autorità religiose le possibili intersezioni tra insegnamenti confessionali e non confessionali della religione e tra questi e l'educazione alla cittadinanza democratica; Régis Debray, L'en seignement du fait religieux dansl'école laïque, Odile Jacob, Paris 2002; cf. anche il documento Le dialogue entre les peuples et les cultures dans l'espace euro-méditerranéen, prodotto dal "Groupe des Sages créé à l'initiative du président de la Commission européenne ", Office des publications officielles des Communautés européennes, Lussemburgo 2004. Sul presente e l'avvenire degli insegnamenti relativi ai tre monoteismi, si veda l'indagine empirica condotta dalla Herbert-Quandt-Stiftung, European identity and cultural pluralism: Judaism, Christianity and Islam in European curricula. Recommendations, Bad Homburg v.d.Höhe 2003.

Sui problemi specifici della formazione degli insegnanti di religione, oltre ai titoli citati nelle note e agli articoli in dizionari specializzati (Diccioná- 
rio de Catequetica, a cura di J.Gevaert; Lexikon des Religionspädagogik, a cura di N.Mette e F. Rickers), si veda: Ulrich Hemel, Introduzione alla Pedagogia religiosa, Queriniana, Brescia 1990; Teódulo García Regidor, La educación religiosa en la escuela, Ediciones San Pio X, Madrid s.d., pp. 161-183; Bernadette Wiame, Pour uneinculturation del"enseignementreligieux, Lumen Vitae, Bruxelles 1997; Mario Cimosa (ed.), L'insegnante di religione nell'attuale rinnovamento dell'educazione scolastica, Las, Roma 1998; Autori Vari, Le cours de religion dans un espace démocratique, in "Lumen Vitae", 2001, n.2; Carlos Esteban Garcés, Enseñanza de la religión y Ley de Calidad, PPC, Madrid 2003, pp.217-261.

Per concludere, si consiglia di visitare i numerosi websites creati in questi anni per appoggiare e promuovere la formazione iniziale e permanente degli insegnanti di religione: consultare i siti nazionali o regionali delle chiese, degli uffici scuola delle diocesi, delle associazioni professionali degli insegnanti, di istituti superiori di scienze religiose e di facoltà di teologia e pedagogia.

\section{Referenciale}

ARTACHO, LOPEZ, Rafael. La formación del Profesor de religión para un nuevo Sistema Educativo. Sinite, n. 97, p. 333-358, 1991.

BADER, Günther. Das ${ }^{\circledR}$ - ein marchenzeichen? Zur pädagogik an religionspädagogischen instituten. Christlichpädagogische Blätter, v.114, n. 3, p. 168-173, 2001.

BAUMANN, Ulrike. Islam in der fordbildung von religionslehrerinnen und religionslehrern. In: Zeitschrift für Pädagogik und Theologie, v. 55, n. 2, p.181-194, 2003.

BERG Horst Klaus. Ausbildung im Spannungsfeld von Hermeneutik und Didaktik. Religions: pädagogische Beiträge, n. 42, p. 67-84,1999.

CAMPOLEONI, Alberto. Insegnanti di religione: materiali per la preparazione del concorso a cattedra. Torino: Editrice SEI, 2003.

COMISION EPISCOPAL DE ENSEÑANZA Y CATEQUESIS. El profesor de religión: Identidad y misión. Madrid: Edice, 1998.

CRAVOTTA, Giovanni. La professionalità docente dell'insegnante di religione cattolica. Insegnare religione, v.12, n. 3, p. 4-18, 1999.

DAMIANO Elio-MORANDI R. (edd.). Cultura Religione Scuola: L'insegnamento della religione nella trasformazione culturale e nell'innovazione scolastica, in prospettiva europea. Franco Angeli editore, Milano, 2000. p. 189-223. 
DEMEL, Sabine. Warum Kirchenrecht für Religionslehrer/innen? Plädoyer für eine ungeliebte, aber unentbehrliche Disziplin der Theologie. Religionspädagogische Beiträge, n. 52, p. 41-48, 2004.

ENGLISH, Leona. M. Mentoring in religious education. Birmingham (Alabama): Religiuos Education, 1998.

GARCIA REGIDOR, Teódulo (ed.). Presente y futuro del profesor de religión: numero monográfico di Sinite, n. 97, p. 223-358, 1991.

HILPERT, Konrad. Selbständiges theologisches Denken, Lehren und Lernen: an Schule und Universität. Religionsunterricht an höheren Schulen, v. 49, n. 4, p. 246-258, 1999.

KUBERA, Ursula. Beruf Religionspädagogin: Aktuelle Problemanzeige und Perspektiven. Religionspädagogische Beiträge, n. 43, p. 121-134,1999.

JENDORFF, Bernhard. Kursus zur Verbesserung des Lehrverhaltens angehender Religionslehrer und-lehrerinnen. Religionspädagogische Beiträge, n. 47, p. 99-110.

LONGO, Giuseppe. Appunti per la formazione degli insegnanti: Alcune riflessioni di ordine psico-pedagogico. Insegnare religione, v.16, n. 3, p.4-9, 2003.

MARENBACH, Udo. Religionspädagogische Kompetenz aus der Sicht eines Studienleiters. Religionspädagogische Beiträge, n. 42, p. 137-149.

PAJER, Flavio. La formazione degli insegnanti di religione in Europa. In: E. DAMIANO (ed.). Riscolarizzare: Atti del convegno nazionale. Provincia autonoma di Trento: Trecento, 1995. p. 294-304

PAJER, Flavio. L'insegnante di religione, una professionalità mortificata. In: G.MALIZIA-Z.Trenti(edd.). Una disciplina al bivio. Torino: Sei, 1996. p. 107-118.

PAJER, Flavio. Stimoli e prospettive per la formazione dell'insegnante di religione dal contesto europeo. In: M.CIMOSA (ed.). L'insegnante di religione nell'attuale rinnovamento dell'educazione scolastica. Roma: Las editrice, 1998. p.11-24.

PRENNA, Lino. Gli Istituti di scienze religiose nella Chiesa: una ipotesi di statuto epistemologico. In: Assicurata ma facoltativa. La religione incompiuta, a cura di L.Prenna. Roma: Ave, 1997. p. 171-178.

RODRIGUEZ, C. Baldomero. El profesor de religión: claves y criterios de valoración sobre su formación, su tarea docente y su vinculación a la Iglesia. Actualidad Catequética, v.38, n 18, p. 600-627,1998. 
SARNATARO, Ciro.; ESPOSITO, Domenico. (edd.). L'Insegnante di religione nella scuola materna, elementare e secondaria. Napoli: Simone, 2004.

SCABINI, Pino. (ed.). Scienze umane e scienze religiose: programmazione e prospettive nell'Istituto di scienze religiose. Roma: Dehoniane, 1989.

SCHLÜTER, Richard. Zur Elementarisierung theologischer Ausbildung und theologischer Persönlichkeitsbildung in neuen kirchlichen Verlautbarungen. Religionspädagogische Beiträge, n. 47, p. 3-10, 2001,

SCHMID, Kuno. Religionsdidaktische Standards in der Lehrerinnen- und Lehrerbildung. Religionspädagogische Beiträge, n.52, p. 3-20, 2004.

TZSCHETZSCH, Werner. Religionslehrer sein: Herausforderungen und Kompetenzen. Theologische Quartal-schrift, v. 792, p. 100-109, 1999.

ZWERGEL, Herbert. A. Zur Forderung kommunikativer Kompetenzen in der Ausbildung. Religionspädagogische Beiträge, n. 42, p. 151-166,1999. 